\title{
COVID-19 and increasing demand for medical oxygen: can impurity be a problem?
}

\author{
Vivek Singh Malik $^{1} \cdot$ Khaiwal Ravindra ${ }^{2} \cdot$ Meenu Singh ${ }^{1}$ \\ Received: 26 August 2021 / Accepted: 2 September 2021 / Published online: 17 September 2021 \\ (C) The Author(s), under exclusive licence to Springer-Verlag GmbH Germany, part of Springer Nature 2021
}

\section{Introduction}

The COVID-19 pandemic in 2021 has accelerated the demand for oxygen supplies globally. Many countries have not seen such high demand before the pandemic to meet their daily oxygen requirement (Usher 2021). Worldwide, governments are scaling up their existing facilities to fill the oxygen demand. In the healthcare system, oxygen is an essential medicine required at all levels. It is estimated that $20-40 \%$ of deaths due to pneumonia could be prevented using oxygen therapy (WHO 2021). Medical air in hospital settings is widely used for mechanical ventilation, infant resuscitation, aerosol drug delivery, and neonatal environment control (Edwards et al. 2018).

Oxygen cylinders, oxygen concentrators, liquid oxygen, and membrane separator oxygen enrichers are commonly used for oxygen therapy (Rees and Dudley 1998). In the current COVID-19 pandemic, long-term oxygen therapy is required at both hospitals and in-home settings that the respiratory physician should supervise for optimal treatment (Rees and Dudley 1998). Unprecedented production of oxygen and refilling of cylinders can give rise to the risk of impurities, which could lead to an increase in hidden mortality. However, it would be difficult to assess the increase in mortality due to the impurity of the oxygen gas. Hence, the purity of the oxygen needs to be regulated by the respective authorities (Edwards et al. 2018). Higher oxygen production can give rise to a possibility of contamination.

Responsible Editor: Lotfi Aleya

Meenu Singh

meenusingh4@gmail.com

1 Department of Pediatrics, Post Graduate Institute of Medical Education and Research (PGIMER), Chandigarh 160012, India

2 Department of Community Medicine and School of Public Health, Post Graduate Institute of Medical Education and Research (PGIMER), Chandigarh 160012, India
In a recent study, the $\mathrm{CO}_{2}$ impurity was reported in oxygen cylinders above the recommended threshold level (Edwards et al. 2018). In previous studies, various health effects were reported due to the common air pollutants (WHO 2005). It was also observed that unintentional inhalation of NO in industrial regions might change the $\mathrm{PaO}_{2}$ (Benzing et al. 1999). Edwards et al. (2018) also mentioned onsite production of medical air poses a risk of raising the $\mathrm{CO}_{2}$ and $\mathrm{NOx}$ (nitric oxides) concentration above the United States Pharmacopeia (USP) threshold (Edwards et al. 2018). This rise of these trace gases may be due to the dryer that is used to capture the $\mathrm{CO}_{2}$ contaminant and humidity.

Impurity sources can be arising due to the production and delivery systems, as well as from the environmental pollution levels near the regions. According to the FDA guidelines, medical gases should be checked for any adulteration during production, packaging, delivery, and manufacturing facilities (FDA 2015). Here, we would like to highlight few points for policymakers/local authorities (Table 1) and for the general public (Table 2) to create awareness and recommendations to improve the quality of oxygen.

Another reason for the increase in the COVID-19 mortality can also be due to the black fungus, which are present in the environment (Richardson 2009). This could lead to mucormycosis, which could be a fatal disease for people who have weakened immune systems (Spellberg et al. 2005). In the nasal tract, black fungus develop due to the poor quality of water used to hydrate the oxygen in a hospital setting (piped and cylinders). This fungus grows in the nostril, travels towards the eyes (Klotz et al. 2000), and then towards the brain, which can paralyze the nerves (Escobar and Del Brutto 1990), cause permanent damage to the eye, or even could lead to a heart attack (Naik et al. 2021; Jackman and Simonsen 1992).

An evidence-based advisory for screening, diagnosis, and management of mucormycosis developed by Cornely et al. (2019) should be followed and need to be updated considering the recent evidence of mucormycosis in the COVID- 
Table 1 Interventions required by policymakers or local authorities

Monitor gas testing facilities, laboratories, and calibration facilities at the site

To ensure compliance and procedures of finished gas for its quality

Purity and grade (ultra-pure, $99.999 \%$ minimum) should be maintained

A random check of tankers and especially cylinders for chemical analysis of oxygen gases

Impurity parameters certificate should be provided for the levels (i.e., carbon monoxide/carbon dioxide, moisture, arsenic, oil, halogen, oxidizing substances, acidity or alkalinity, argon, hydrocarbons)

Test results can be cross-checked by the local authority for any foreign materials, moisture, and contaminants

To monitor any misuse of other cylinders (i.e., helium, hydrogen, acetylene, argon) for refilling oxygen without proper cleaning and conditioning

Monitoring for the sanitization of oxygen supply (pipeline and tubes) for unwanted fungal growth

Special attention should be given to test "black fungus" (mucormycosis) infection among recovered patients

Checking/advisory for black fungus on patients on oxygen support should be made an essential part of the COVID-19 control protocol at every level

Special attention/care should be given to comorbid and patients using the steroid to check the fungal infection for the hidden cause of death

19 pandemic (Cornely et al. 2019). In a hospital setting, constantly monitor distilled water for oxygen hydration before using it in oxygen therapy devices (Cahill and Heath 1990). Use of tap water even after boiling is not recommended in the humidifier as after some time; impurities (micro-metals and minerals/salts) start to buildup, leading to severe health issues even after using life-saving precious oxygen therapy.

Table 2 Recommendation/awareness needed for the general public

Oxygen gas cylinder should be kept at normal room temperature Always use distilled water to make oxygen hydrated before use

Never use poor-quality water such as tap water, boiled water, or purified RO water

Masks, nasal cannulae should be cleaned and checked for any leakage by the attendee

If using an oxygen concentrator, attendee should adhere to manufacturer guidelines for proper cleaning as and when required

The oxygen concentrator should be placed in a well-ventilated and clean area

Oxygen filters should be properly cleaned from time to time

Avoid smoking (wood, cigarette, sticks) in any form, active or passive, near to oxygen concentrator

Attendee can monitor patient nostril for any black pigmentation, as soon as it is noticed even as the smallest spot contact doctor immediately for further treatment

Avoid irrational use of steroids without recommendations
Globally, we are facing an emergency that needs support from authorities and the community to minimize the suffering and improve the well-being of all. In lower- and middleincome countries, oxygen purity can be a significant issue that requires local interventions at the earliest. Countries where there is prevalence of malaria, sepsis, pneumonia, and other ailments should also prioritize where oxygen therapy requires at large (Usher 2021).

Author contribution Vivek Singh Malik, Khaiwal Ravindra, and Meenu Singh all contributed equally in writing, reviewing, and final editing of the manuscript.

Data availability Not applicable.

\section{Declarations}

Ethical approval Not applicable.

Consent to participate Not applicable.

Consent to publish Not applicable.

Competing interests The authors declare that they have no competing interests.

\section{References}

Benzing A, Loop T, Mols G, Geiger K (1999) Unintended inhalation of nitric oxide by contamination of compressed air. Anesthesiology 91(4):945-950. https://doi.org/10.1097/00000542-19991000000013

Cahill CK, Heath J (1990) Sterile water used for humidification in lowflow oxygen therapy: is it necessary? Am J Infect Control 18(1):1317. https://doi.org/10.1016/0196-6553(90)90205-7

Cornely OA, Alastruey-Izquierdo A, Arenz D, Chen SCA, Dannaoui E, Hochhegger B, Hoenigl M et al (2019) Global guideline for the diagnosis and management of mucormycosis: an initiative of the European Confederation of Medical Mycology in Cooperation with the Mycoses Study Group Education and Research Consortium. Lancet Infect Dis 19(12):e405-e421. https://doi.org/10.1016/ S1473-3099(19)30312-3

Edwards P, Therriault P-A, Katz I (2018) Onsite production of medical air: is purity a problem? Multidiscip Respir Med 13(1):12. https:// doi.org/10.1186/s40248-018-0125-8

Escobar A, Del Brutto OH (1990) Multiple brain abscesses from isolated cerebral mucormycosis. J Neurol Neurosurg Psychiatry 53(5):431433. https://doi.org/10.1136/jnnp.53.5.431

FDA (2015) “7356-002E Compressed Medical Gases," 12/03. https:// www.fda.gov/media/75194/download. Accessed 15 Aug 2021

Government of Canada CC for OH and S, CAN/CSA-Z7396-1-2009 (2009) Medical gas pipeline systems - part 1: pipelines for medical gases and vacuum. https://www.lokring.com/wp-content/uploads/ Approvals/CSA-Z7396-1-2009.pdf

Jackman JD, Simonsen RL (1992) The clinical manifestations of cardiac mucormycosis. Chest 101(6):1733-1736. https://doi.org/10.1378/ chest.101.6.1733 
Klotz SA, Penn CC, Negvesky GJ, Butrus SI (2000) Fungal and parasitic infections of the eye. Clin Microbiol Rev 13(4):662-685. https://doi. org/10.1128/CMR.13.4.662

Naik CA, Mathai SK, Sandkovsky US, Ausloos KA, Guileyardo JM, Schwartz G, Mason DP, Gottlieb R, Grazia TJ (2021) Acute myocardial infarction secondary to mucormycosis after lung transplantation. IDCases 23:e01019. https://doi.org/10.1016/j.idcr.2020. $\mathrm{e} 01019$

Rees PJ, Dudley F (1998) ABC of oxygen: provision of oxygen at home. BMJ 317(7163):935-938. https://doi.org/10.1136/bmj.317.7163. 935

Richardson M (2009) The ecology of the zygomycetes and its impact on environmental exposure. Clin Microbiol Infect 15:2-9. https://doi. org/10.1111/j.1469-0691.2009.02972.x

Spellberg B, Edwards J, Ibrahim A (2005) Novel perspectives on mucormycosis: pathophysiology, presentation, and management.
Clin Microbiol Rev 18(3):556-569. https://doi.org/10.1128/CMR. 18.3.556-569.2005

Usher AD (2021) Medical oxygen crisis: a belated COVID-19 response. Lancet 397(10277):868-869. https://doi.org/10.1016/S01406736(21)00561-4

WHO (2005) Air quality guidelines - global update. http://www.who.int/ phe/health_topics/outdoorair/outdoorair_aqg/en/. Accessed 24 Aug 2021

WHO (2021) "The-Life-Saving-Power-of-Medical-Oxygen." https:// www.who.int/news-room/feature-stories/detail/the-life-savingpower-of-medical-oxygen. Accessed 24 Aug 2021

Publisher's note Springer Nature remains neutral with regard to jurisdictional claims in published maps and institutional affiliations. 